\title{
COMPOSIÇÃO QUÍMICA E VALORES DE ENERGIA METABOLIZÁVEL DE INGREDIENTES ALTERNATIVOS PARA FRANGOS DE CORTE
}

\author{
Marcos José Batista dos SANTOS ${ }^{1}$, MARIA CARMo MOHAUPT MARQUES LUdKE ${ }^{2}$, JORGE VitOR \\ LUDKE $^{3}$, THAYSA RODRIGUES TORRES ${ }^{4}$, LETÍCIA DOS SANTOS LOPES ${ }^{5}$, MARIANY SOUZA BRITO ${ }^{6}$ \\ ${ }^{1}$ Pós graduando da Universidade Federal Rural de Pernambuco, Recife, PE, Brasil - \\ marcoszootecnista@gmail.com \\ ${ }^{2}$ Professores Doutores da Universidade Federal Rural de Pernambuco, Recife. PE, Brasil \\ ${ }^{3}$ Pesquisador Doutor da EMBRAPA Suínos e Aves, Concórdia, SC, Brasil. \\ ${ }^{4}$ Pós-graduanda da Universidade Federal Rural de Pernambuco, Recife. PE, Brasil. \\ ${ }^{5}$ Analista da EMBRAPA Suínos e Aves, Concórdia, SC, Brasil. \\ ${ }^{6}$ Pós-graduanda da Universidade Federal de Paraíba, Bananeiras, PB, Brasil.
}

Um experimento foi realizado para determinar a composição química e os valores de energia metabolizável aparente (EMA) e EMA corrigida para nitrogênio (EMAn) de três tipos de farelo de algodão (FA): FA extrusado, FA com extração por solvente com casca e FA com $40 \%$ de proteína bruta (PB); duas variedades de sorgo e farelo residual de milho (FRM). Valores de EMAn do FA e do sorgo da literatura foram usados para estabelecer equações de predição através da composição nutricional. Pintos de corte machos Ross $(n=210)$ foram alojados com seis repetições por tratamento e cinco aves por gaiola. Foi utilizada uma ração-referência (RR) e seis rações-teste contendo os ingredientes a avaliar em substituição de 40 $\%$ da RR. A composição química dos ingredientes foi:
92,$92 ; 91,80 ; 89,08 ; 86,44 ; 87,19 ; 88,50 \%$ de matéria seca; 32,$96 ; 35,11 ; 40,50 ; 9,83 ; 8,17$ e $10,80 \%$ de PB, 17,$81 ; 1,03 ; 3,59 ; 2,27 ; 2,67$ e $12,90 \%$ de extrato etéreo (EE) e os valores de EMAn foram 2977, 2793, 2827, 2766,3117 e $3017 \mathrm{kcal} / \mathrm{kg}$ para o FA extrusado, FA com casca, FA com 40\% PB, sorgo IPA, sorgo Dow e FRM, respectivamente. A equação de EMAn para o FA foi $9158,67+1106,94 * \mathrm{~PB}-12,05 * \mathrm{~PB} * \mathrm{~PB}-1866,99 *$ Cinzas $(\mathrm{CZ})+100,16 * \mathrm{CZ} * \mathrm{CZ}-834,01 *$ Fibra Bruta $(\mathrm{FB})+$ $30,43 * \mathrm{FB} * \mathrm{FB}$ e para o sorgo foi $4365,59+175,41 * \mathrm{~PB}-$ $10,35 * \mathrm{~PB} * \mathrm{~PB}-99,55 * \mathrm{EE}+525,34 * \mathrm{CZ}-85,06 * \mathrm{CZ} * \mathrm{CZ}-$ $1310,47 * \mathrm{FB}+251,61 * \mathrm{FB} * \mathrm{FB}$. Os valores de EMAn de uma variedade de sorgo e um tipo de FA não se ajustaram às equações de predição estabelecidas.

PALAVRAS-CHAVE: equações de predição; farelo de algodão; farelo residual de milho; metabolismo; sorgo

\section{CHEMICAL COMPOSITION AND METABOLIZABLE ENERGY VALUES OF ALTERNATIVE INGREDIENTS FOR BROILERS}

\section{ABSTRACT}

An experiment was conducted to determine the chemical composition and apparent metabolizable energy (AME) and AME corrected for nitrogen (AMEn) of three types of cottonseed meal (CM): CM extruded, $\mathrm{CM}$ by solvent extraction with husk and CM with $40 \%$ crude protein (CP), two varieties of sorghum and hominy feed. AMEn values of $\mathrm{CM}$ and sorghum from the existing literature were used to create prediction equations using chemical composition. Male Ross broilers at 14 days of age $(n=210)$ were used in a metabolism assay and housed in metabolism cages in groups of five animals with six replicates per treatment. A reference diet (RD) and six test diets containing the ingredients under evaluation replacing $40 \%$ of RD constituted the set of established treatments. The chemical composition of the ingredients was as follows: $92.92,91.80,89.08,86.44,87.19$ and $88.50 \%$ for dry matter, $32.96,35.11,40.50,9.83,8.17$ and $10.80 \%$ for $\mathrm{CP}, 17.81,1.03,3.59,2.27,2.67$ and $12.90 \%$ for ether extract (EE) and the values of AMEn were 2977, 2793, 2827, 2766, 3117 and $3017 \mathrm{kcal} / \mathrm{kg}$, respectively, for 
extruded CM, CM with hulls, CM with $40 \% \mathrm{CP}$, IPA sorghum, Dow sorghum and hominy feed. The established AMEn equation for CM was $-9,158.67+1,106.94 * \mathrm{CP}$ $12.05 * \mathrm{CP} * \mathrm{CP}-1,866.99 * \mathrm{Ash}+100.16^{*} \mathrm{Ash} * \mathrm{Ash}-$ $834.01 *$ Crude Fiber $(\mathrm{CF})+30.43 * \mathrm{CF} * \mathrm{CF}$ and for sorghum was $4,365.59+175.41 * \mathrm{CP}-10.35 * \mathrm{CP} * \mathrm{CP}-$ 99.55*EE + 525.34*Ash $-85.06 *$ Ash*Ash $-1,310.47 * \mathrm{CF}$ $+251.61 * \mathrm{CF} * \mathrm{CF}$. AMEn values of one sorghum and one $\mathrm{CM}$ type did not adjust to the prediction equations established.

KEYWORDS: cotton seed meal; hominy feed; metabolism; prediction equations; sorghum.

\section{INTRODUÇÃO}

$\mathrm{Na}$ avicultura brasileira, os gastos com alimentação representam cerca de 60 a $75 \%$ dos custos totais, sendo que o milho e o farelo de soja compõem a maior parte do custo da ração. A falta de informações técnicas limita, ou mesmo impede, a utilização de alimentos alternativos (MURAKAMI et al., 2009) e, por isso, promover estudos para tornar possível a substituição, parcial ou total, dos ingredientes mais onerosos de forma econômica é um fator que contribui para a viabilização da produção.

O farelo de algodão (FA) é um ingrediente em potencial, podendo ser usado como fonte protéica nas rações para frangos de corte em condições de baixo (OJEWOLA et al., 2006), médio (PIMENTEL et al., 2007) e alto desempenho (SANTOS et al., 2008). O uso para aves é restringido pela limitação em lisina (AZMAN \& YILMAZ, 2005), elevado teor de fibra bruta e pela presença do gossipol, mas, torna-se viável quando associado a outros ingredientes protéicos a fim de melhorar o valor da proteína da ração e substituir fontes protéicas de origem animal. Os subprodutos resultantes do processamento da semente de algodão para extração de óleo representam, com uma disponibilidade relativa de $6 \%$, a terceira mais importante fonte protéica de oleaginosa para a alimentação animal no mundo (NAGALAKSHMI et al., 2007; USDA, 2011). No Brasil, a estimativa de produção atual é de 3,2 milhões de toneladas de caroço de algodão (CONAB, 2011). A forma de processamento aplicada ao caroço de algodão determina diversos tipos de farelo no mercado que se diferenciam entre si no teor de proteína, óleo residual e fibra (WALDROUP \& KERSEY, 2002).

O sorgo é uma gramínea relevante para regiões semi-áridas pelas suas características xerófilas com maior possibilidade de adaptação que o milho às condições adversas de menor disponibilidade de água e temperatura diurna elevada (SANCHEZ et al., 2002). A produção estimada de sorgo em grão (CONAB, 2011) é de 2,3 milhões de toneladas, o que representa cerca de $4,4 \%$ da produção anual de milho no Brasil. O grão tem características nutricionais semelhantes ao milho em termos de energia metabolizável (SEDGHI et al., 2011), com um teor maior em proteína bruta dependendo do ambiente e da fertilidade do solo (DOUGLAS et al., 1990;
ANTUNES et al., 2006). Como desvantagem, apresenta menores teores de alguns aminoácidos (arginina, glicina e histidina) e pigmentos do que o milho (GARCIA et al., 2005) e apresenta compostos fenólicos, kafirina e fitatos. A kafirina tem correlação negativa com a digestibilidade dos aminoácidos e a energia metabolizável verdadeira corrigida para retenção de nitrogênio (EMVn) (BRYDEN et al., 2009). Adicionalmente, a digestibilidade dos aminoácidos e dos carboidratos no trato digestivo das aves é menor e mais lenta do que a do milho em função do complexo proteína:carboidrato no endosperma, comprometendo a conversão alimentar de frangos de corte (BRYDEN et al., 2009).

$\mathrm{O}$ farelo residual de milho (FRM) resulta como subproduto da industrialização do milho destinado ao consumo humano. Esse ingrediente gerado no processamento a seco corresponde como subproduto a um terço do milho industrializado e é composto por casca, gérmen e porções de amido extraídos do grão, devendo apresentar um mínimo de $4 \%$ de óleo (BRUM et al., 2000). Segundo cálculos a partir de estatísticas (ABIMILHO, 2011), a disponibilidade anual estimada de FRM no país é de 1,1 milhão de toneladas, sendo tradicionalmente utilizado na alimentação de gado leiteiro. Porém, com o uso mais generalizado de enzimas nas dietas de frangos de corte (ADEOLA et al., 2010), também existe uma expectativa para a substituição parcial do milho pelo FRM (KACZMAREK et al., 2009).

Esses ingredientes alternativos têm potencial uso na avicultura de corte, porém sua inclusão nas rações ainda é avaliada como de risco pelos produtores devido a incertezas sobre o valor nutricional. Sendo assim, esse experimento foi conduzido com os seguintes objetivos: a) determinar a composição química e a energia metabolizável aparente (EMA) e aparente corrigida para nitrogênio (EMAn) do FRM, de duas variedades de sorgo e de três tipos de FA oriundos de diferentes processamentos e b) estabelecer equações de predição para a EMAn do sorgo e FA mediante o emprego dos resultados obtidos e dados de literatura.

\section{MATERIAL E MÉTODOS}

$\mathrm{O}$ experimento foi desenvolvido no laboratório de pesquisa em metabolismo de Não 
Ruminantes do Departamento de Zootecnia da Universidade Federal Rural de Pernambuco (DZ/UFRPE). Amostras dos ingredientes utilizados grão de sorgo de duas variedades, farelo residual de milho (FRM) e farelos de algodão (FA) submetidos a diferentes processamentos (FA extrusado, FA com casca e FA $40 \%$ de proteína bruta) - foram coletadas e analisadas no laboratório de Nutrição Animal do DZ/UFRPE. As amostras foram analisadas quanto aos teores de matéria seca (MS), proteína bruta $(\mathrm{PB})$, extrato etéreo (EE), fibra bruta $(\mathrm{FB})$, cinzas $(\mathrm{CZ})$, cálcio, fósforo e energia bruta utilizando-se as metodologias descritas por SILVA \& QUEIROZ (2002). O cálculo do Extrativo Não Nitrogenado (ENN), expresso na base matéria natural, foi realizado utilizando-se a fórmula:

$\mathrm{ENN}=100-[(100-\mathrm{MS})-\mathrm{CZ}-\mathrm{FB}-\mathrm{PB}-\mathrm{EE}]$.

Posteriormente, desenvolveu-se um ensaio de metabolismo, utilizando-se pintos de corte machos da linhagem Ross, para determinação da energia metabolizável aparente (EMA) e da EMA corrigida para balanço de nitrogênio (EMAn) dos ingredientes. O método utilizado foi o de coleta total das excretas, conforme descrito por HILL \& ANDERSON (1958), utilizando-se o fator de correção $8,22 \mathrm{kcal} / \mathrm{g}$ para retenção de nitrogênio. Os valores de EMA e EMAn dos ingredientes foram determinados por meio das equações propostas por MATTERSON et al. (1965).

Duzentos e dez pintos de um dia foram alojados em baterias metálicas, cada uma composta por seis gaiolas dentro de uma sala de metabolismo climatizada, sob regime de iluminação contínua (durante 24 horas/dia) e temperatura média de 23,5 \pm $2,8^{\circ} \mathrm{C}$. As gaiolas eram providas de bebedouros tipo copo e comedouros tipo calha e dotadas de bandejas cobertas com plásticos, dispostas de modo a coletar as excretas por unidade experimental. Aos 14 dias de idade as aves com peso médio de $396 \pm 10 \mathrm{~g}$ foram distribuídas em sete tratamentos com seis repetições, em delineamento em blocos casualizados e cinco aves por unidade experimental. Os sete tratamentos consistiram em uma dieta-referência à base de milho e farelo de soja, conforme apresentado na Tabela 1, e seis dietas-teste constituídas de $60 \%$ da dietareferência e $40 \%$ do ingrediente em avaliação. A dieta-referência foi formulada com composição nutricional obtida a partir de tabela padrão (ROSTAGNO et al., 2011).

A água e as rações fareladas foram fornecidas à vontade. O período de adaptação das aves às dietas foi de cinco dias, seguido de mais cinco dias para coleta total de excretas. Como marcador fecal para determinar o início e o final da coleta de excretas, foi utilizado o óxido férrico em pó, foi homogeneizado às rações experimentais na proporção de $0,1 \%$. As excretas foram coletadas duas vezes ao dia em dois horários, às oito e 16 horas. A cada coleta, procedeuse o acondicionamento do material em sacos plásticos, sendo devidamente identificados e armazenados em freezer a uma temperatura de $-20{ }^{\circ} \mathrm{C}$ até o final do experimento, quando as amostras foram homogeneizadas por unidade experimental. Amostras representativas das rações e das excretas foram enviadas ao laboratório para realização das análises laboratoriais de matéria seca, proteína bruta e energia bruta.

Tabela 1. Composição da ração referência ${ }^{1}$

\begin{tabular}{|c|c|}
\hline Ingredientes & $\%$ \\
\hline Milho grão moído (peneira 2mm) & 59,34 \\
\hline Farelo de soja & 34,36 \\
\hline Óleo de soja & 2,29 \\
\hline Fosfato bicálcico & 1,8 \\
\hline Calcário calcítico & 0,89 \\
\hline Sal comum iodado & 0,49 \\
\hline DL-Metionina (99\%) & 0,24 \\
\hline L-Lisina $(78,8 \%)$ & 0,19 \\
\hline Premix vitamínico $^{2}$ e micro-mineral ${ }^{3}$ & 0,40 \\
\hline Total & 100,00 \\
\hline \multicolumn{2}{|c|}{ 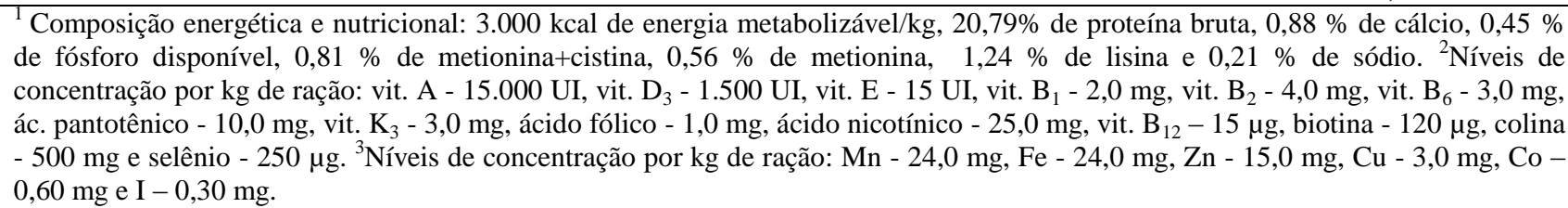 } \\
\hline
\end{tabular}


Equações de predição da EMAn do sorgo e do FA foram estabelecidas utilizando-se dados publicados em tabelas de composição de alimentos nacionais e internacionais. Para realizar as análises de regressão linear múltipla de predição, foram utilizados os componentes lineares e quadráticos dos valores de composição química $(\mathrm{EE}, \mathrm{FB}, \mathrm{PB}, \mathrm{CZ})$ expressos na base matéria seca. As análises de regressão foram realizadas utilizando-se o método Stepwise de Eliminação Indireta (Backward) e o SAS (2008). Em cada equação, foram mantidas as variáveis com efeitos significativos, a $5 \%$ de probabilidade pelo teste $\mathrm{T}$ e, quando o componente quadrático foi significativo, o componente linear também foi mantido na equação. $\mathrm{O}$ uso dos valores de ENN nas equações foi restringido para reduzir efeitos de colinearidade. Após o estabelecimento das equações iniciais com a seleção das variáveis preditoras, para o conjunto de dados inicial que gerou a equação, procedeu-se à comparação do valor predito com o valor determinado no ensaio de metabolismo ou valor tabelado. Uma vez definida a equação, pelos critérios de $\mathrm{R}^{2}$ ajustado e do teste $\mathrm{T}$ para os coeficientes estimados, ainda foram adotados critérios adicionais de restrição com base na amplitude da diferença entre valores reais e preditos. Como critério, foi estabelecido que a diferença entre esses valores para os ingredientes não pode ser maior do que $1 \%$ do valor de EMAn nas rações formuladas (de 28 a $32 \mathrm{kcal}$ ), quando da inclusão do nível ótimo no caso de ingrediente energético e do que $0,5 \%$ (de 14 a $16 \mathrm{kcal}$ ) no caso de ingrediente proteico. Assim, para considerar que determinada fonte de dados pudesse fazer parte da análise final para predição, foram adotados critérios de restrição de desvio máximo entre predito e real de 50 kcal (considerando $56 \%$ de inclusão na ração para frangos de corte) para o sorgo e de $75 \mathrm{kcal}$ (18\% de inclusão na ração) para o FA.

\section{RESULTADOS E DISCUSSÃO}

Os farelos de algodão avaliados têm características nutricionais peculiares, que são consequência dos métodos de processamento. Conforme apresentado na Tabela 2, o FA extrusado apresenta baixo teor relativo de $\mathrm{FB}(7,56 \%)$, devido à retirada da casca, e alto teor de EE (17,81\%), em função da perda de uma pequena fração do óleo durante a extrusão; assim, o teor de PB $(32,96 \%)$ fica intermediário entre o teor presente no caroço de algodão não processado e aquele presente no farelo de alta proteína. O FA com casca obtido via extração com solvente apresenta uma concentração de EE de apenas $1,03 \%$ e teor de FB de 15,94\% em função da adição parcial da casca. O FA com alta proteína, no valor de $40,5 \%$, apresenta um teor de FB de $11,9 \%$ resultante da adição parcial de casca após a extração do óleo por prensa helicoidal ("screw-press"). No processo ainda persiste um teor de óleo residual e a concentração de EE é de 3,59\%. Esse FA apresenta concentração de FB e de PB semelhante à relatada por EMBRAPA (1991); porém, o valor de EE é duas vezes superior. O FA com casca apresenta concentrações de FB e de EE próximas aos relatados por ROSTAGNO et al. (2005; 2011), mas, nessa comparação, a concentração de PB é inferior.

Como regra geral, a concentração de EE na torta de algodão, oriunda da extração mecânica via prensa hidráulica, situa-se entre $4 \%$ e $8 \%$. Entretanto, quando oriunda de processamento em prensa de eixo helicoidal, a torta de algodão mantém teores de EE entre $3 \%$ e $5 \%$. Na extração por solvente, o farelo apresenta menos de $3 \%$ de óleo residual, sendo que o valor geralmente fica próximo a $1 \%$. WALDROUP \& KERSEY (2002) relataram a composição nutricional do farelo ou torta de algodão obtidos via extração por solvente ou extração mecânica oriundos de 16 fábricas dos EUA e especificam que, em função de diferentes manejos, a maior variabilidade ocorre nas concentrações de FB e de EE. Nos FA sob avaliação no presente trabalho, o teor máximo de gossipol total detectado foi de $0,12 \%$.

Tabela 2. Composição bromatológica do farelo de algodão (FA) extrusado, FA com extração por solvente com casca e FA $40 \%$ de proteína bruta (PB), sorgo IPA, sorgo Dow e farelo residual de milho com os valores de matéria seca (MS), proteína bruta $\mathrm{PB}$, extrato etéreo (EE), fibra bruta (FB), cinzas (CZ), cálcio (Ca) e fosforo (P), expressos em porcentagem e energia bruta (EB) expressa em $\mathrm{kcal} / \mathrm{kg}$ na base matéria natural

\begin{tabular}{lcccccccc}
\hline Ingrediente & MS & PB & EE & FB & CZ & EB & Ca & P \\
\hline FA extrusado & 92,92 & 32,96 & 17,81 & 7,56 & 4,80 & 5365 & 0,11 & 0,78 \\
FA com casca & 91,80 & 35,11 & 1,03 & 15,94 & 5,02 & 4287 & 0,10 & 0,76 \\
FA 40\% PB & 89,08 & 40,50 & 3,59 & 11,90 & 5,54 & 4655 & 0,12 & 0,94 \\
Sorgo IPA & 86,44 & 9,83 & 2,27 & 1,76 & 1,07 & 3903 & 0,02 & 0,20 \\
Sorgo Dow & 87,19 & 8,17 & 2,67 & 1,79 & 0,72 & 3829 & 0,01 & 0,23 \\
Farelo residual de milho & 88,50 & 10,80 & 12,90 & 5,04 & 3,90 & 4638 & 0,01 & 0,85 \\
\hline
\end{tabular}


Os grãos de sorgo granífero avaliados pertencem ao grupo genético I (isento de tanino): IPA $7301011^{\circledR}$ de coloração branca com tanino abaixo de $0,10 \%$ e Dow Agroscience $822^{\circledR}$ de coloração vermelha, sendo ambas as variedades com testa (subcamada abaixo do pericarpo) sem pigmentação. O sorgo Dow apresentou um teor de PB menor do que o sorgo IPA, além de menor PB do que os valores tabelados por ROSTAGNO et al. (2005; 2011) e NRC (1994). A FB, o EE e a CZ nos sorgos DOW e IPA estavam em menor concentração do que os valores tabelados em NRC (1994) e ROSTAGNO et al. (2011). O valor de EB do sorgo IPA é semelhante ao valor tabelado por ROSTAGNO et al. (2011), enquanto o sorgo Dow tem uma concentração de cerca de $100 \mathrm{kcal} / \mathrm{kg}$ menor ao valor tabelado.

O FRM apresentou uma composição nutricional semelhante àquela descrita para o farelo de gérmen de milho (ROSTAGNO et al., 2011). Os valores de PB e FB foram similares aos descritos pelo NRC (1994); contudo, o EE foi muito superior aos valores publicados por NRC (1994) e BRUM et al. (2000).

Os valores de EMAn calculados, em média, foram inferiores em $4,84 \%$ aos valores de EMA (Tabela 3). Essa diferença caracteriza a amplitude da retenção de nitrogênio pelas aves. A correção pelo balanço de nitrogênio tem por objetivo padronizar e reduzir a variação nos valores de EMA dos ingredientes medidos e aplicados em diferentes condições (LOPEZ \& LEESON, 2008). Segundo esses autores, os valores de EMA e EMAn para o milho são pouco afetados pela idade dos frangos e a correção para retenção de nitrogênio foi consistente em 3\%. Os sorgos avaliados apresentaram uma elevada diferença entre si, o que potencialmente está relacionado com as diferenças no teor de PB. Os coeficientes de metabolização da energia $(\mathrm{CME}=$ $100 *$ EMAn/EB) sofrem influência do tipo de processamento aplicado nos diferentes FA e os valores calculados são superiores aos apresentados por ROSTAGNO et al. (2011), que variam de 40,3 a $46,7 \%$ para FA oriundos por extração por solvente com alta $(24,93 \%)$ e baixa $(13,97 \%)$ concentração de FB. Entretanto, os valores se aproximam do valor $58,8 \%$ para FA com teor de $12,96 \%$ de FB estabelecido por BRUMANO et al. (2006) para frangos de corte com média de idade de 45 dias. O CME para o FA extração por solvente com baixo teor de FB $(11,98 \%)$ calculado a partir de EMBRAPA (1991) é de 52,5\%.

O valor de CME do sorgo Dow é semelhante aos que podem ser calculados em NAGATA et al. (2004), com $80 \%$ para grão moído e $81 \%$ para grão inteiro; em NUNES et al. (2008), com $81,1 \%$ e ROSTAGNO et al. (2011), com $81,5 \%$. Esses valores de CME contrastam com o calculado para o sorgo IPA que está mais próximo ao valor calculado $(74,8 \%)$ a partir dos dados de BRUM et al. (2000). Para o FRM, o valor de CME calculado está próximo ao relatado $(69,0 \%)$ por BRUM et al. (2000); no entanto, é inferior aos valores calculados $(72,7 \%) \mathrm{em}$ NAGATA et al. (2004) e $(74,0 \%)$ em ROSTAGNO et al. (2011) para o gérmen de milho.

O valor de $2977 \mathrm{kcal}$ de EMAn/kg determinado para o FA extrusado é superior aos valores estabelecidos em tabela nacional (ROSTAGNO et al., 2011) e internacional (NRC, 1994), que apresentam 1947 e 1657 kcal $/ \mathrm{kg}$, respectivamente. Entretanto, deve-se considerar que a concentração de EE do FA extrusado é muito superior aos valores de EE dos FA apresentados nas tabelas e que a retirada da casca permite um baixo valor de FB, bem inferior aos valores tabelados.

BRUMANO et al. (2006) estabeleceram o valor de $2461 \mathrm{kcal}$ de EMAn/kg para o FA quando realizaram um ensaio de metabolismo utilizando frangos de corte com idade entre 41 e 50 dias, valor este significativamente diferente daquele determinado para a idade entre 21 e 30 dias (1963 $\mathrm{kcal} / \mathrm{kg}$ ). SHARMA et al. (1978) determinaram um valor de $2601 \mathrm{kcal}$ de EMAn/kg para um FA com $89 \%$ de MS, $12,5 \%$ de FB e 7,4\% de EE, originado por extração controlada via prensa. O FA com 40\% PB com valor de EMAn de $3173 \mathrm{kcal} / \mathrm{kg}$ de MS não apresentou o ajuste esperado à equação apresentada na Tabela 4. Dos fatores que contribuem para as oscilações nos valores energéticos nos ensaios de metabolismo, existem aqueles relacionados aos alimentos: composição físico-química, processamentos, digestibilidades e fatores antinutricionais; aos animais: genética, idade e sexo e, aos metodológicos: tempo de duração do ensaio, nível de inclusão do alimento, composição da dieta referência, taxa de consumo e ao ambiente (SOARES et al., 2005; LOPEZ \& LEESON, 2008; ADEOLA \& ILELEJI, 2009). 
Tabela 3. Média dos valores de matéria seca (MS), do coeficiente de metabolização (CME), energia metabolizável aparente (EMA) e EMA corrigida para o nitrogênio (EMAn) de três farelos de algodão (FA), dois sorgos e do farelo residual de milho (FRM)

\begin{tabular}{lccccc}
\hline Ingrediente & MS $(\%)$ & CME $(\%)$ & EMA (kcal/kg) & $\begin{array}{c}\text { EMAn } \\
(\mathrm{kcal} / \mathrm{kg})\end{array}$ & $\begin{array}{c}\text { Diferença (\%) EMA } \\
\text { e EMAn }\end{array}$ \\
\hline FA Extrusado & 92,92 & 55,5 & $3131 \pm 58,2$ & $2977 \pm 60,4$ & 4,92 \\
FA com casca & 91,80 & 65,2 & $2944 \pm 27,7$ & $2793 \pm 22,1$ & 5,13 \\
FA com 40\% PB & 89,08 & 60,7 & $3016 \pm 59,2$ & $2827 \pm 72,2$ & 6,27 \\
Sorgo IPA & 86,44 & 70,9 & $2908 \pm 44,1$ & $2766 \pm 45,3$ & 4,88 \\
Sorgo Dow & 87,19 & 81,4 & $3206 \pm 15,3$ & $3117 \pm 64,3$ & 2,78 \\
FRM & 88,50 & 65,0 & $3178 \pm 42,3$ & $3017 \pm 32,2$ & 5,07 \\
\hline
\end{tabular}

Em relação ao sorgo variedade IPA, a EMAn ficou abaixo do valor esperado, não estando de acordo com ROSTAGNO et al. (2011) e NRC (1994), que apresentam valores de 3189 e 3288 $\mathrm{kcal} / \mathrm{kg}$, respectivamente. O resultado de EMAn para a variedade dow está próximo ao citado nessas tabelas. NAGATA et al. (2004) determinaram os valores de 3137 e 3177 kcal de EMAn/kg, respectivamente, para o sorgo moído e inteiro, apresentando $88,90 \%$ de MS. No entanto, BRUM et al. (2000) apresentaram o valor de $2954 \mathrm{kcal}$ de EMAn/kg para o sorgo baixo tanino. O sorgo IPA com EMAn no valor de $3200 \mathrm{kcal} / \mathrm{kg}$ de MS apresentou falta de ajuste à equação apresentada na tabela 4. Existem fatores intrínsecos aos grãos dos diferentes tipos de sorgo, principalmente na textura do endosperma (ANTUNES et al., 2006), que interferem na digestibilidade do amido (BENMOUSSA et al., 2006) e da proteína (DUODU et al., 2003), afetando seu efetivo valor nutricional para frangos de corte (KRIEGSHAUSER et al., 2006; SALINAS et al., 2006). Fatores ambientais, tais como déficit hídrico na época do enchimento dos grãos, podem afetar o valor nutricional, o que invalida a comparação entre variedades diferentes produzidas em condições climáticas diferentes.

Tabela 4. Equações para estimativa do valor de energia metabolizável aparente corrigida para retenção de nitrogênio (EMAn) expressas com base em matéria seca para o sorgo e farelo de algodão

\begin{tabular}{|c|c|c|c|c|c|}
\hline \multirow{2}{*}{$\begin{array}{l}\text { Ingrediente } \\
\text { Componente }\end{array}$} & \multirow[b]{2}{*}{ Parâmetro* } & \multicolumn{2}{|c|}{ Sorgo $(n=19)$} & \multicolumn{2}{|c|}{ Farelo de Algodão $(\mathrm{n}=13)$} \\
\hline & & Coeficiente & Prob & Coeficiente & Prob \\
\hline \multirow{3}{*}{ Proteína Bruta } & Constante & 4365,59 & $<0,0001$ & $-9158,67$ & 0,0013 \\
\hline & $\mathrm{L}$ & 175,41 & 0,0744 & 1106,94 & $<0,0001$ \\
\hline & Q & $-10,35$ & 0,0182 & $-12,05$ & $<0,0001$ \\
\hline \multirow{2}{*}{ Extrato Etéreo } & $\mathrm{L}$ & $-99,55$ & 0,0008 & - & - \\
\hline & Q & - & - & - & - \\
\hline \multirow{2}{*}{ Cinza } & $\mathrm{L}$ & 525,34 & 0,0002 & $-1866,99$ & $<0,0001$ \\
\hline & $\mathrm{Q}$ & $-85,06$ & 0,0036 & 100,16 & $<0,0001$ \\
\hline \multirow{2}{*}{ Fibra Bruta } & $\mathrm{L}$ & $-1310,47$ & $<0,0001$ & $-834,01$ & $<0,0001$ \\
\hline & Q & 251,61 & $<0,0001$ & 30,43 & $<0,0001$ \\
\hline \multirow{2}{*}{\multicolumn{2}{|c|}{ Prob. do modelo de regressão }} & \multicolumn{2}{|c|}{$\mathrm{p}<0,0001$} & \multicolumn{2}{|c|}{$\mathrm{p}<0,0001$} \\
\hline & Coeficiente de determinação & \multicolumn{2}{|c|}{0,9619} & \multicolumn{2}{|c|}{0,9908} \\
\hline \multicolumn{2}{|c|}{ Erro de predição média (kcal) } & \multicolumn{2}{|c|}{19,63} & \multicolumn{2}{|c|}{22,41} \\
\hline \multicolumn{2}{|c|}{ Erro de predição máximo (kcal) } & \multicolumn{2}{|c|}{44,8} & \multicolumn{2}{|c|}{75,5} \\
\hline \multicolumn{2}{|c|}{$\begin{array}{l}\text { Diferença entre estimado e } \\
\text { determinado** }\end{array}$} & \multicolumn{2}{|c|}{$31 \mathrm{kcal}$} & \multicolumn{2}{|c|}{$5 \mathrm{kcal}(1)$ e $13 \mathrm{kcal}(2)$} \\
\hline
\end{tabular}

*L= Linear, Q= Quadrático. Os parâmetros que multiplicam os coeficientes têm valores que são expressos em base matéria seca. A probabilidade apresentada refere-se a cada um dos parâmetros significativos. **Sorgo Dow com valor de EMAn de $3575 \mathrm{kcal} / \mathrm{kg}$ de MS e FA com valores de EMAn determinados de (1) $3204 \mathrm{kcal} / \mathrm{kg}$ de MS (FA extrusado) e (2) $3042 \mathrm{kcal} / \mathrm{kg}$ de MS (FA c/ casca). 
O valor calculado de EMAn para o FRM está próximo ao relatado $(3040 \mathrm{kcal} / \mathrm{kg})$ por BRUM et al. (2000), porém é inferior ao valor $(3144 \mathrm{kcal} / \mathrm{kg}$ ) apresentado por ROSTAGNO et al. (2011) para o gérmen de milho e superior ao valor $(2896 \mathrm{kcal} / \mathrm{kg})$ apresentado no NRC (1994) para o "hominy feed". Ao adotar a equação EMAn $=3839,5+53,80 \times$ EE $264,46 \times$ MM, proposta por NASCIMENTO et al. (2009), para estimar os valores de EMAn para alimentos concentrados energéticos para aves, o valor estimado de energia para o FRM é de 3060 $\mathrm{kcal} / \mathrm{kg}$ (na base natural), com uma diferença de 43 $\mathrm{kcal} / \mathrm{kg}$, representando 1,4\%. ROCHELL et al. (2011) consideram que o uso de EB, PB e fibra em detergente neutro como parâmetros para estimar subprodutos do milho geram estimativas mais precisas.

O coeficiente de correlação de Pearson entre a concentração de $\mathrm{PB}$ e o valor de EMAn do sorgo é significativo e indica que à medida que o nível de $\mathrm{PB}$ aumenta, o valor de EMAn diminui (Tabela 5). Esse resultado corrobora os obtidos por BRYDEN et al. (2009), que caracterizaram correlação negativa entre EMVn e nível de PB no sorgo, que, segundo os autores, é condicionada pela formação de complexos de baixa digestibilidade entre a kafirina e carboidratos. Para o FA, os coeficientes de correlação de Pearson que foram significativos para a EMAn foram o EE, correlacionado de forma positiva, e a $\mathrm{CZ}$ que foi correlacionada de forma negativa.

Tabela 5. Coeficiente de correlação de Pearson e os níveis de significância entre os componentes da composição nutricional e a EMAn para o sorgo e o farelo de algodão

\begin{tabular}{lcccc}
\hline Ingrediente & \multicolumn{2}{c}{ Sorgo $(\mathrm{n}=19)$} & \multicolumn{2}{c}{ Farelo de Algodão $(\mathrm{n}=13)$} \\
\hline & EMAn & $\mathrm{p}=$ & EMAn & $\mathrm{p}=$ \\
\hline Proteína bruta & $-0,5621$ & 0,0123 & $-0,1561$ & 0,6105 \\
Extrato etéreo & 0,0859 & 0,7266 & 0,609 & 0,0272 \\
Cinzas & 0,2109 & 0,3862 & $-0,5449$ & 0,0542 \\
Fibra bruta & 0,3879 & 0,1008 & $-0,3888$ & 0,1892 \\
\hline
\end{tabular}

Quando os resultados das Tabelas 4 e 5 são considerados de forma conjunta, é possível interpretar que a $\mathrm{PB}$, a $\mathrm{FB}$ e a $\mathrm{CZ}$, de forma associada com seus componentes lineares e quadráticos na equação de predição da EMAn para o FA, conseguem explicar o efeito isolado que teria o EE.

\section{CONCLUSÕES}

Valores de EMAn determinados para os farelos de algodão avaliados são diferentes dos referenciados na literatura, sendo reflexo de diferenças na tecnologia de processamento adotada, alterando a composição nutricional. No sorgo, a variedade IPA tem valor de EMAn que fica abaixo do esperado, o que representa a situação do grão produzido sob condição de estresse hídrico. A equação de EMAn estabelecida para o FA foi $9158,67+1106,94 * \mathrm{~PB}-12,05 * \mathrm{~PB} * \mathrm{~PB}-$ $1866,99 * \mathrm{CZ}+100,16 * \mathrm{CZ} * \mathrm{CZ}-834,01 * \mathrm{FB}+$ $30,43^{*} \mathrm{FB} * \mathrm{FB}$ e para o sorgo foi $4365,59+$ $175,41 * \mathrm{~PB}-10,35^{*} \mathrm{~PB} * \mathrm{~PB}-99,55^{*} \mathrm{EE}+$ $525,34 * \mathrm{CZ}-85,06 * \mathrm{CZ} * \mathrm{CZ}-1310,47 * \mathrm{FB}+$ $251,61 * \mathrm{FB} * \mathrm{FB}$. O uso de equações de predição fundamentadas na composição nutricional e nas restrições impostas não permite estimar a EMAn para uma variedade de sorgo e um tipo de FA avaliados.

\section{REFERÊNCIAS}

ABIMILHO - ASSOCIAÇÃO BRASILEIRA DAS INDÚSTRIAS DE MILHO. Estatística - Consumo por processo. Disponível em: www.abimilho.com.br. Acesso: 12 de outubro de 2011.

ADEOLA, O.; ILELEJI, K. E. Comparision of two diet types in the determination of metabolizable energy content of corn distillers dried grains with solubles for broiler chickens by the regression method. Poultry Science, v.88, n. 3, p. 579-585, 2009.

ADEOLA, O.; JENDZA, J. A.; SOUTHERN, L. L.; POWELL, S.; OWUSU-ASIEDU, A. Contribuition of exogenous dietary carbohidrases to the metabolizable energy value of corn distillers grains for broiler chickens. Poultry Science, v. 89, n. 7, p. 1947-1954, 2010.

ANTUNES, R. C.; RODRIGUEZ, N. M.; GONÇALVES, L. C.; RODRIGUES, J. A. S.; BAIÃO, N. C.; PEREIRA, L. G. R.; LARA, L. J. Valor nutritivo de grãos de sorgo com diferentes texturas do endosperma para frangos de corte. Arquivos Brasileiros de Medicina Veterinária e Zootecnia, v. 58, n. 5, p. 877-883, 2006.

AZMAN, M. A.; YILMAZ, M. The growth performance 
of broiler chicks fed diets containing cottonseed meal supplemented with lysine. Revue Medecine Veterinaire, v. 156, n. 2, p. 104-106, 2005

BENMOUSSA, M.; SUHENDRA, B.; ABOUBACAR, A.; HAMAKER, B. R. Distinctive sorghum starch granule morphologies appear to improve raw starch digestibility. Starch-Stärke, v. 58, n. 2, p. 92-99, 2006.

BRUM, P. A. R.; ZANOTTO, D. L.; LIMA, G. J. M. M.; VIOLA, E. S. Composição química e energia metabolizável de ingredientes para aves. Pesquisa Agropecuária Brasileira, v. 35, n. 5, p. 995-1002, 2000.

BRUMANO, G.; GOMES, P. C.; ALBINO, L. F. T.; ROSTAGNO, H. S.; GENEROSO, R. A. R.; SCHMIDT, M. C. Composição química e valores de energia metabolizável de alguns alimentos protéicos determinados com frangos de corte em diferentes idades. Revista Brasileira de Zootecnia, v. 35, n. 6, p. 2297-2302, 2006.

BRYDEN, W. L.; SELLE, P. H.; CADOGAN, D. J.; LI, X.; MULLER, N. D.; JORDAN, D. R.; GIDLEY, M. J.; HAMILTON, W. D. A review of the nutritive value of sorghum for broilers. RIRDC Publication $\mathrm{N}^{\circ}$ 9/007, 68 p., 2009.

CONAB - COMPANHIA NACIONAL DE ABASTECIMENTO. Acompanhamento da safra brasileira: Primeiro levantamento da produção de grãos safra 2011/2012. Outubro de 2011. CONAB, Brasília, 2011, 40 p. Disponível em www.conab.gov.br. Acesso: 12 de outubro de 2011.

DOUGLAS, J. H.; SUlLIVAN, T. W.; BOND, P. L.; STRUWE, F. J. Nutrient composition and metabolizable energy values of selected grain sorghum varieties and yellow corn. Poultry Science, v. 69, n. 6, p. 1147-1155, 1990.

DUODU, K.; TAYLOR, J. R. N.; BELTON, P. S.; HAMAKER, B. R. Factors affecting sorghum protein digestibility. Journal of Cereal Science, v. 38, n. 2, p.117-131, 2003 .

EMPRESA BRASILEIRA DE PESQUISA AGROPECUÁRIA - EMBRAPA. Centro Nacional de Pesquisa de Suínos e Aves. Tabela de composição química e valores energéticos de alimentos para suínos e aves. 3 ed. Concórdia, SC: EMBRAPA - CNPSA, Série Documentos, n. 19, 97 p., 1991

GARCIA, R. G.; MENDES, A. A.; COSTA, C.; PAZ, I. C. L. A.; TAKAHASHI, S. E.; PECÍlIA, K. P.; KOMIYAMA, C. M.; QUINTEIRO, R. R. Desempenho e qualidade da carne de frangos de corte alimentados com diferentes níveis de sorgo em substituição ao milho. Arquivo Brasileiro de Medicina Veterinária e Zootecnia, v. 57, n. 5, p. 634-643, 2005.

HILL, F. W.; ANDERSON, D. L. Comparation of metabolizable energy and productive energy determination with growing chicks. The Journal of Nutrition, v. 64, n. 4, p. 587-608, 1958.

KACZMAREK, S.; BOCHENEK, M.; JÓZEFIAK, D.;
RUTKOWSKI, A. Effect of enzyme supplementation of diets based on maize or hominy feed on performance and nutrient digestibility in broilers. Journal of Animal and Feed Sciences, v. 18, n. 2, p. 113-123, 2009.

KRIEGSHAUSER, T. D.; TUINSTRA, M. R.; HANCOCK, J. D. Variation in nutritional value of sorghum hybrids with contrasting seed weight characteristics and comparisions with maize in broiler chicks. Crop Science, v. 46, n. 2, p. 695-699, 2006.

LOPEZ, G.; LEESON, S. Assessment of the nitrogen correction factor in evaluating metabolizable energy of corn and soybean meal in diets for broilers. Poultry Science, v. 87, n. 2, p. 298-306, 2008.

MATTERSON, L. D.; POTTER, L. M.; STUTS, M. W.; SINGSEN, E. P. The metabolizable energy of feed ingredients for chickens. Storrs: Connecticut, the University of Connecticut. Agricultural Experiment Station, Research Report, n. 7, 1965, 11p.

MURAKAMI, A. E.; SOUZA, L. M. G.; MASSUDA, E. M.; ALVES, F. V.; GUERRA, R. H.; GARCIA, A. F. Q. Avaliação econômica e desempenho de frangos de corte alimentados com diferentes níveis de milheto em substituição ao milho. Acta Scientiarum. Animal Sciences, v. 31, n. 1, p. 31-37, 2009.

NAGALAKSHMI, D.; RAO, S. V. R.; PANDA, A. K.; SASTRY, V. R. B. Cotton meal in poultry diets: A Review. The Journal of Poultry Science, v. 44, n. 2, p.119-134, 2007.

NAGATA, A. K.; RODRIGUES, P. B.; FREITAS, R. T. F.; BERTECHINI, A. G.; FIALHO, E. T. Energia metabolizável de alguns alimentos energéticos para frangos de corte, determinada por ensaios metabólicos e por equações de predição. Ciência e Agrotecnologia, v.8, n. 3, p. 668-677, 2004.

NASCIMENTO, G. A. J.; RODRIGUES, P. B.; FREITAS, R. T. F.; BERTECHINI, A. G.; LIMA, R. R.; PUCCI, L. E. A. Equações de predição para estimar valores da energia metabolizável de alimentos energéticos para aves utilizando meta-análise. Revista Brasileira de Zootecnia, v. 38, n. 7, p. 1265-1271, 2009.

NATIONAL RESEARCH COUNCIL. Nutrient Requirements of Poultry. Ninth Revised Edition, Washington D.C.: National Academy Press, 1994, 155 p.

NUNES, R. V.; ROSTAGNO, H. S.; GOMES, P. C.; NUNES, C. G. V.; POZZA, P. C.; ARAUJO, M. S. Coeficientes de metabolizabilidade da energia bruta de diferentes ingredientes para frangos de corte. Revista Brasileira de Zootecnia, v. 37, n. 1, p. 89-94, 2008.

OJEWOLA, G. S.; UKACHUKWU, S. N.; OKULONYE, E. I. Cottonseed meal as substitute for soyabean meal in broiler ration. International Journal of Poultry Science, v. 5, n. 4, p. 360-364, 2006.

PIMENTEL, A. C. S.; DUTRA JÚNIOR, W. M.; LUDKE, M. C. M. M.; LUDKE, J. V.; RABELLO, C. B. V., FREITAS, C. R. G. Substituição parcial do milho e 
farelo de soja por sorgo e farelo de caroço de algodão extrusado em rações de frangos de corte. Acta Scientiarum. Animal Sciences, v. 29, n. 2, p. 135-141, 2007.

ROCHELL, S. J.; KERR, B. J.; DOZIER III, W. A. Energy determination of corn co-products fed to broiler chicks from 15 to 24 days of age, and use of composition analysis to predict nitrogen-corrected apparent metabolizable energy. Poultry Science, v. 90, n. 10, p.1999-2007, 2011.

ROSTAGNO, H. S.; ALBINO, L. F. T.; DONZELE, J. L.; GOMES, P. C.; OLIVEIRA, R. F.; LOPES, D. C.; FERREIRA, A. S.; BARRETO, S. L. T. Tabelas brasileiras para aves e suínos: composição de alimentos e exigências nutricionais de aves e suínos. $2^{\mathrm{a}}$ edição, Viçosa, MG: UFV, 186 p., 2005.

ROSTAGNO, H. S.; ALBINO, L. F. T.; DONZELE, J. L.; GOMES, P. C.; OLIVEIRA, R. F.; LOPES, D. C.; FERREIRA, A. S.; BARRETO, S. L. T.; EUCLIDES, R. F. Tabelas brasileiras para aves e suínos: composição de alimentos e exigências nutricionais de aves e suínos.

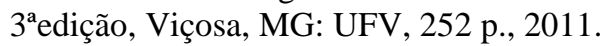

SALINAS, I.; PRÓ, A.; SALINAS, Y.; SOSA, E.; BECERRIL, C. M.; CUCA, M.; CERVANTES, M.; GALEGOS, J. Compositional variation amongst sorghum hybrids: Effect of Kafirin concentration on metabolizable energy. Journal of Cereal Science, v. 44, n. 3, p. 342-346, 2006.

SANCHEZ, A. C.; SUBUDHI, P. K.; ROSENOW, D. T.; NGUYEN, H. T. Mapping QTLs associated with drought resistance in sorghum (Sorghum bicolor L. Moench). Plant Molecular Biology, v. 48, n. 5-6, p. 713-726, 2002.
SANTOS, A. P. S. F.; LUDKE, M. C. M. M.; LUDKE, J. V.; FRAIHA, M.; OLIVEIRA, E. L.; TORRES, T. R.; SANTOS, M. J. B.; VILELA, M. R. O. Carcass traits of broilers feed with cottonseed meal. Archivos de Zootechnia, v. 57, n. 217, p. 15-24, 2008.

SAS INSTITUTE INC. System for Microsoft Windows, Release 9.2 - Standard Edition, Cary, NC, USA, 20022008. (CD-ROOM).

SEDGHI, M.; EBADI, M. R.; GOLIAN, A.; AHMADI, $\mathrm{H}$. Estimation and modeling true metabolizable energy of sorghum grain for poultry. Poultry Science, v. 90, n. 5, p.1138-1143, 2010.

SHARMA, N. K.; LODHI, G. N.; ICHHPONANI, J.S. Comparative feeding value of expeller-processed undecorticated and decorticated cottonseed cakes for growing chicks. Journal of Agricultural Science, v. 91, n. 3 , p. 531-541, 1978.

SILVA, D. J.; QUEIROZ, A. C. Análise de alimentos: métodos químicos e biológicos. 3.ed. Viçosa, MG: Editora UFV, 235 p., 2002.

SOARES, K. R.; BERTECHINI, A. G.; FASSANI, E. J.; RODRIGUES, P. B.; FIALHO, E. T.; GERALDO, A.; BRITO, J. A. G. Valores de energia metabolizável de alimentos para pintos de corte na fase pré-inicial. Ciência e Agrotecnologia, v. 29, n. 1, p. 238-244, 2005.

USDA - UNITED STATES DEPARTMENT OF AGRICULTURE. Oilseeds: World markets and trade. USDA Foreign Agricultural Service: Circular Series, FOP 10-11, 33 p., 2011.

WALDROUP, P. W.; KERSEY, J. H. Nutrient composition of cottonseed meal surveyed. Feedstuffs, v.74, n. 45 , p. 11-12, 2002.

Protocolado em: 27 mar. 2012. Aceito em: 30 nov. 2012. 